\title{
The graph of a totally geodesic foliation
}

\author{
by Robert A. Wolak (Kraków)
}

\begin{abstract}
We study the properties of the graph of a totally geodesic foliation. We limit our considerations to basic properties of the graph, and from them we derive several interesting corollaries on the structure of leaves.

In this short note we study the properties of the graph of a totally geodesic foliation. Its importance comes from the fact that the graph of a foliation is the starting point of the construction of the $C^{*}$-algebra associated with this foliation. The value of $C^{*}$-algebras in the study of foliations cannot be overestimated, but this goes beyond the scope of this note. We limit our considerations to basic properties of the graph, and from them we derive several interesting corollaries on the structure of leaves.

The definition and the basic properties of the graph of a foliation can be found in [8]. Let us recall the definition.

The graph $\operatorname{GR}(\mathcal{F})$ of the foliation $\mathcal{F}$ is the space of equivalence classes of triples $(y, \alpha, x)$ where $x$ and $y$ are points of the same leaf $L$ of $\mathcal{F}$ and $\alpha$ is a path in $L$ linking $x$ to $y$. Two triples $(y, \alpha, x)$ and $\left(y^{\prime}, \alpha^{\prime}, x^{\prime}\right)$ are equivalent iff $x=x^{\prime}, y=y^{\prime}$ and the holonomy of the curve $\alpha^{-1} * \alpha^{\prime}$ is trivial. A neighbourhood of $\langle y, \alpha, x\rangle$ consists of elements represented by the triples of the form $\left(y^{\prime}, \alpha^{\prime}, x^{\prime}\right)$ where $x^{\prime}$ belongs to some neighbourhood of $x$ in a transverse manifold passing through $x, y^{\prime}$ belongs to some neighbourhood of $y$ in a transverse manifold passing through $y$, and $\alpha^{\prime}$ is the holonomy lift of $\alpha$ to $x^{\prime}$ (see [8]). In the same paper it is proved that the graph of a foliation is a manifold of dimension $n+p$ but in general non-Hausdorff. Moreover, if the elements of the holonomy pseudogroup are determined by their jets then the graph is a Hausdorff topological space.
\end{abstract}

The mappings

$$
p_{1}:\langle y, \alpha, x\rangle \mapsto x
$$

1991 Mathematics Subject Classification: Primary 53C12, 57R30.

Key words and phrases: foliation, totally geodesic, graph. 
the source projection, and

$$
p_{2}:\langle y, \alpha, x\rangle \mapsto y,
$$

the target projection, define two submersions $p_{1}: \operatorname{GR}(\mathcal{F}) \rightarrow M$ and $p_{2}$ : $\operatorname{GR}(\mathcal{F}) \rightarrow M$. In local coordinates they can be written as follows. Let $\langle y, \alpha, x\rangle$ be a point of $\operatorname{GR}(\mathcal{F})$ and $(U, \varphi),(V, \psi)$ and $\left(U \times_{\alpha} V, \varphi \times_{\alpha} \psi\right)$ be adapted charts at $x, y$ and $\langle y, \alpha, x\rangle$, respectively, where

$$
\begin{array}{r}
U \times{ }_{\alpha} V=\left\{\left\langle y^{\prime}, \alpha^{\prime}, x^{\prime}\right\rangle \in \operatorname{GR}(\mathcal{F}): x^{\prime} \in U, \varphi\left(x^{\prime}\right)=\left(x_{1}, x_{2}\right) \in \mathbb{R}^{p} \times \mathbb{R}^{q},\right. \\
y^{\prime} \in V, \psi\left(y^{\prime}\right)=\left(y_{1}, y_{2}\right) \in \mathbb{R}^{p} \times \mathbb{R}^{q}, h_{\alpha}\left(x_{2}\right)=y_{2} \\
\text { and } \left.\alpha^{\prime} \text { is the holonomy lift of } \alpha \text { to } x^{\prime}\right\}
\end{array}
$$

and

$$
\varphi \times{ }_{\alpha} \psi(\langle y, \alpha, x\rangle)=\left(x_{1}, x_{2}, x_{3}\right) \in \mathbb{R}^{p} \times \mathbb{R}^{q} \times \mathbb{R}^{p}
$$

Then

$\varphi \circ p_{1} \circ\left(\varphi \times_{\alpha} \psi\right)^{-1}: \mathbb{R}^{p} \times \mathbb{R}^{q} \times \mathbb{R}^{p} \rightarrow \mathbb{R}^{p} \times \mathbb{R}^{q}, \quad\left(x_{1}, x_{2}, x_{3}\right) \mapsto\left(x_{1}, x_{2}\right)$,

and

$\psi \circ p_{1} \circ\left(\varphi \times{ }_{\alpha} \psi\right)^{-1}: \mathbb{R}^{p} \times \mathbb{R}^{q} \times \mathbb{R}^{p} \rightarrow \mathbb{R}^{q} \times \mathbb{R}^{p}, \quad\left(x_{1}, x_{2}, x_{3}\right) \mapsto\left(x_{3}, h_{\alpha}\left(x_{2}\right)\right)$.

On $\operatorname{GR}(\mathcal{F})$ there are three foliations:

(i) $\mathcal{F}_{1}$ defined by the fibres of $p_{1}$;

(ii) $\mathcal{F}_{2}$ defined by the fibres of $p_{2}$;

(iii) $\mathcal{F}_{1} \oplus \mathcal{F}_{2}=p_{1}^{-1} \mathcal{F}=p_{2}^{-1} \mathcal{F}$.

The fibres of the submersions $p_{1}$ and $p_{2}$ are the holonomy coverings of leaves of $\mathcal{F}$. Let $Q$ be a subbundle of $T M$ complementary to $T \mathcal{F}$ and $\widetilde{Q}$ be the subbundle of $T \operatorname{GR}(\mathcal{F})$ defined as follows:

$$
\widetilde{Q}=\left\{X \in T \operatorname{GR}(\mathcal{F}): d p_{1}(X) \in Q, d p_{2}(X) \in Q\right\} .
$$

The tangent bundle of $\operatorname{GR}(\mathcal{F})$ admits the following decomposition:

$$
T \operatorname{GR}(\mathcal{F})=T \mathcal{F}_{1} \oplus T \mathcal{F}_{2} \oplus \widetilde{Q}
$$

A curve $\gamma$ in $\operatorname{GR}(\mathcal{F})$ is tangent to $\widetilde{Q}$ iff the curves $p_{1} \gamma$ and $p_{2} \gamma$ are tangent to $Q$. Moreover, the fibre bundle $\widetilde{Q}$ is isomorphic to $p_{1}^{*} Q$ and $p_{2}^{*} Q$, and $\widetilde{Q}=p_{1}^{-1} Q \cap p_{2}^{-1} Q$.

Using the method developed in $[11,12]$ and the fact that totally geodesic foliations have the property of lifting paths (see $[5,2]$ ), we can easily prove the following:

THEOREM 1. Let $\mathcal{F}$ be a transversely analytical totally geodesic foliation of a complete Riemannian manifold $M$. Then the projections $p_{1}: \operatorname{GR}(\mathcal{F}) \rightarrow$ $M$ and $p_{2}: \operatorname{GR}(\mathcal{F}) \rightarrow M$ are locally trivial fibre bundles. Their fibres are the holonomy coverings of leaves. 
In [9] Winkelnkemper proved that in such a situation leaves have at most two ends, i.e.

COROLlARY 1. Let $\mathcal{F}$ be a transversely analytical totally geodesic foliation of a complete simply connected Riemannian manifold $M$. Then leaves of $\mathcal{F}$ have one or two ends.

Now we turn our attention to geometrical properties of the graph. The subbundle $T \mathcal{F}_{1} \oplus \widetilde{Q}$ of $T \operatorname{GR}(\mathcal{F})=T \mathcal{F}_{1} \oplus T \mathcal{F}_{2} \oplus \widetilde{Q}$ is isomorphic to $p_{2}^{*} T M$, and $T \mathcal{F}_{2} \oplus \widetilde{Q}$ to $p_{1}^{*} T M$. These isomorphisms provide us with two natural ways of defining a Riemannian metric on $\operatorname{GR}(\mathcal{F})$. Let us describe one of them.

On $T \mathcal{F}_{2} \oplus \widetilde{Q} \cong p_{1}^{*} T M$ we set $\bar{g}=p_{1}^{*} g$, where $g$ is the Riemannian metric on $M$. We make $T \mathcal{F}_{1}$ orthogonal to $T \mathcal{F}_{2} \oplus \widetilde{Q}$. The bundle $T \mathcal{F}_{1}$ is isomorphic to $p_{2}^{*} T \mathcal{F}$. Thus on this subbundle we take $\bar{g}=p_{2}^{*} g \mid T \mathcal{F}$. This completes the definition of a Riemannian metric on $\operatorname{GR}(\mathcal{F})$.

Our aim is to demonstrate that:

If $\mathcal{F}$ is totally geodesic for the Riemannian metric $g$ of $M$, then $\mathcal{F}_{1}$ is totally geodesic for the Riemannian metric $\bar{g}$ of $\operatorname{GR}(\mathcal{F})$.

Therefore we will have to prove that

$$
\left(L_{X} \bar{g}\right)(Y, Z)=0
$$

for any vector field $X$ orthogonal to $\mathcal{F}_{1}$ and vectors $Y, Z$ tangent to $\mathcal{F}_{1}$. As our foliation is defined by a global submersion and the vector field $X$ is a section of $T \mathcal{F}_{2} \oplus \widetilde{Q} \cong p_{1}^{*} T M, X$ can be assumed to be a projectible vector field, i.e. there exists a vector field $\bar{X}$ on $M$ such that $\bar{X}=d p_{1}(X)$ (as $X$ is a section of $p_{1}^{*} T M$ this condition determines the vector field $X$ ). The vectors $Y$ and $Z$ can be extended to vector fields of the form $p_{2}^{*} \bar{Y}$ and $p_{2}^{*} \bar{Z}$, respectively, where $\bar{Y}$ and $\bar{Z}$ are vector fields tangent to $\mathcal{F}$.

First assume that $\bar{X}$ is tangent to $\mathcal{F}$. Then

$$
d p_{1}([X, Y])=0=d p_{2}([X, Y]) .
$$

Thus

$$
L_{X} \bar{g}(Y, Z)=\partial_{X} \bar{g}(Y, Z)
$$

But as $d p_{2}(X)=0$, along any integral curve $\gamma(t)$ of $X$ we have

$$
\bar{g}_{\gamma(t)}(Y, Z)=g_{p_{2} \gamma(t)}(\bar{Y}, \bar{Z})=g_{p_{2} \gamma(0)}(\bar{Y}, \bar{Z}) .
$$

Therefore $\left(L_{X} \bar{g}\right)(Y, Z)=0$ for any vector field $X$ tangent to $T \mathcal{F}_{2}$.

Now consider the case of a vector field $X$ tangent to $\widetilde{Q}$. By restricting our attention to vector fields $X$ defined by vector fields $\bar{X}$ of a special form we can assume that the vector field $X$ is $p_{2}$-projectible as well. The problem is local, thus we can consider the foliation $\mathcal{F}$ defined by a global submersion $f: M \rightarrow N$ with connected fibres. Let $v \in Q_{X}$. Then the vector $d f(v)$ can 
be extended to a global vector field $\widehat{X}$ on $N$, which in turn can be lifted to a global section $\bar{X}$ of $Q$ such that $\bar{X}(x)=v$. Integral curves of the vector field $\bar{X}$ have the following property:

Let $\alpha:[0, l] \rightarrow M$ be a leaf curve and $\gamma:[0, \varepsilon] \rightarrow M$ be the integral curve of $\bar{X}$ with $\gamma(0)=\alpha(0)$. Let $\sigma:[0, l] \times[0, \varepsilon] \rightarrow M$ be the square defined by $(\alpha, \gamma)$. Then the curve $\sigma \mid\{l\} \times[0, \varepsilon]$ is the integral curve of $\bar{X}$ through $\alpha(l)$.

This property can be easily derived from the construction of the square in [1].

Now let $X$ be a $T \mathcal{F}_{2} \oplus \widetilde{Q}$-horizontal lift of $\bar{X}$ to $\operatorname{GR}(\mathcal{F})$. Then the vector field $X$ is a section of $\widetilde{Q}$. We have to show the following:

If $m$ and $m^{\prime}$ are points of an open set of the form $U \times_{\alpha} V$ and $p_{2}(m)=$ $p_{2}\left(m^{\prime}\right)$, then $d p_{2}(X(m))=d p_{2}\left(X\left(m^{\prime}\right)\right)$.

If $d p_{2}(X(m))=d p_{2}\left(X\left(m^{\prime}\right)\right)$, then $m=\langle y, \alpha, x\rangle$ and $m^{\prime}=\left\langle y, \alpha^{\prime}, x^{\prime}\right\rangle$. Thus the points $x$ and $x^{\prime}$ belong to the same plaque and the curve $\alpha^{\prime}$ is the concatenation of a curve $\beta$ linking $x^{\prime}$ to $x$ in the plaque and $\alpha$.

The lifting $X$ of $\bar{X}$ can also be described by the liftings of integral curves of $\bar{X}$. Let $\gamma:(-\varepsilon, \varepsilon) \rightarrow M$ be an integral curve of $\bar{X}$ and $m=\langle y, \alpha, x\rangle$ be a point of $\operatorname{GR}(\mathcal{F})$ over $\gamma(0)=x$. Then the integral curve of $X$ passing through the point $m$ is as follows:

Let $\sigma:[0, l] \times(-\varepsilon, \varepsilon) \rightarrow M$ be the square defined by $(\alpha, \gamma)$. Let $\sigma_{t}$ denote $\sigma \mid[0, l] \times\{t\} ;$ for any $t \in(-\varepsilon, \varepsilon), \sigma_{t}$ is tangent to $\mathcal{F}$. Then

$$
\bar{\gamma}(t)=\left(\sigma(0, t), \sigma_{t}, \sigma(l, t)\right)
$$

is the integral curve of $X$ with $\bar{\gamma}(0)=m$. Let $\gamma^{\prime}:(-\varepsilon, \varepsilon) \rightarrow M$ be the integral curve of $\bar{X}$ with $x^{\prime}=\gamma^{\prime}(0)$. Denote by $\sigma^{\prime}$ the square defined by $\left(\alpha^{\prime}, \gamma^{\prime}\right)$. We have noticed that $\alpha^{\prime}=\alpha * \beta$ for some $\beta:[0, b] \rightarrow M$ and $\gamma^{\prime}=\kappa \mid\{b\} \times(-\varepsilon, \varepsilon)$ where $\kappa$ is the square defined by $\left(\beta^{-1}, \gamma\right)$. Therefore, from the uniqueness of these squares it results that

$$
\sigma_{b+l}^{\prime}=\sigma_{l}
$$

is the integral curve of $\bar{X}$ through $y$. The curve

$$
(-\varepsilon, \varepsilon) \ni t \mapsto\left(\sigma^{\prime}(0, t), \sigma_{t}^{\prime}, \sigma^{\prime}(l+b, t)\right)
$$

is the integral curve of $X$ through $m^{\prime}$ and the curve

$$
\left.(-\varepsilon, \varepsilon) \ni t \mapsto \sigma(0, t), \sigma_{t}, \sigma(l, t)\right)
$$

is the integral curve of $X$ through $m$. Therefore as $\sigma_{b+l}^{\prime}=\sigma_{l}$,

$$
d p_{2}(X(m))=d p_{2}\left(X\left(m^{\prime}\right)\right) .
$$

Thus we have just demonstrated that for our special choice of the vector field $\bar{X}$ the vector field $X$ is $p_{1}$ - and $p_{2}$-projectible. Having proved this fact we can easily complete our calculations. 
Let $X, Y, Z$ be vector fields as previously chosen. Then

$$
L_{X} \bar{g}(Y, Z)=\partial_{X} \bar{g}(Y, Z)-\bar{g}(Y,[X, Z])-\bar{g}([X, Y], Z) .
$$

As $d p_{1}([X, Y])=d p_{1}([X, Z])=0,[X, Y]$ and $[X, Z]$ are tangent to $\mathcal{F}_{1}$. Thus

$$
L_{X} \bar{g}(Y, Z)=\partial_{\hat{X}} g(\bar{Y}, \bar{Z})-g(\bar{Y},[\bar{X}, \bar{Z}])-g([\bar{X}, \bar{Y}], \bar{Z}),
$$

where $\bar{X}, \bar{Y}, \bar{Z}$ are vector fields on $M$ such that $d p_{2}(X)=\bar{X}, d p_{2}(Y)=\bar{Y}$ and $d p_{2}(Z)=\bar{Z}$. Therefore

$$
L_{X} \bar{g}(Y, Z)=L_{\hat{X}} g(\bar{Y}, \bar{Z})=0
$$

as the foliation $\mathcal{F}$ has been assumed to be totally geodesic. By linearity the above equality is true for any vector fields $Y, Z$ tangent to $\mathcal{F}$ and $X$ orthogonal to this foliation. Hence we have proved the following theorem.

THEOREM 2. Let $\mathcal{F}$ be a transversely analytical totally geodesic foliation of a complete Riemannian manifold $M$. Then there exists a Riemannian metric on $\operatorname{GR}(\mathcal{F})$ in which the foliation by fibres of the natural projection onto $M$ is totally geodesic.

Taking into account Theorem 1 we have the following equivalent version of Theorem 2.

THEOREM 3. Let $\mathcal{F}$ be a transversely analytical totally geodesic foliation of a complete Riemannian manifold $M$. There exists a Riemannian metric on $\operatorname{GR}(\mathcal{F})$ for which the structure group of the locally trivial fibre bundle $p_{1}: \operatorname{GR}(\mathcal{F}) \rightarrow M$ can be reduced to a group of isometries.

As a corollary, we recover the well-known fact that leaves of a totally geodesic foliation have isometric universal coverings (see [1]).

One of the most important corollaries of this theorem concerns tangential holonomy, a notion introduced in [2]. Among other things, it is proved there that there exist homomorphisms

$$
\pi_{1}(L, x) \rightarrow H_{g}(L, x) \rightarrow H(L, x),
$$

where $\pi_{1}(L, x)$ is the fundamental group of a leaf $L, H_{g}(L, x)$ is the tangential holonomy group of $L$ at $x$ and $H(L, x)$ is the holonomy group of $L$ at $x$. Both homomorphisms are surjective. In [4] G. Cairns demonstrated that for Riemannian foliations the tangential holonomy group of a leaf is equal to the "standard" holonomy group of this leaf. Theorem 2 ensures that the same is true for transversely analytical totally geodesic foliations.

THEOREM 4. Let $\mathcal{F}$ be a transversely analytical totally geodesic foliation. Then for any leaf $L$ of $\mathcal{F}$ its tangential holonomy group is equal to its holonomy group.

P r o of. We have to show that the homomorphism $H_{g}(L, x) \rightarrow H(L, x)$ is injective. Let $\alpha$ be a loop in the leaf $L$ at $x$ defining the identity in the "germ" 
holonomy group. Let $\gamma$ be any orthogonal curve through $x$. As the holonomy along $\alpha$ is trivial the loop $\alpha$ lifts to a loop at any point $m \in \operatorname{GR}(\mathcal{F})$ over $x$. Denote by $\widetilde{\gamma}$ the $\widetilde{Q}$-lift of $\gamma$ at $m$. The foliation $\mathcal{F}_{1}$ is without holonomy, thus the tangential holonomy group of any leaf is trivial (see [2]), i.e. if $\sigma$ is the square defined by $(\widetilde{\alpha}, \widetilde{\gamma})$ at $m$, then $\sigma_{0}=\sigma_{l}$. The square at $x$ defined by $(\alpha, \gamma)$ is equal to $p_{1} \sigma$. Thus the curve $\alpha$ defines the trivial element in the tangential holonomy group at $x$.

From Theorem 4 we can draw a series of corollaries:

COROLlary 2. If there exists a compact leaf with finite holonomy group then all leaves are compact and have finite holonomy, i.e. the space of leaves is a Hausdorff Satake manifold (orbifold).

Corollary 3. If there exists a leaf with finite volume and finite holonomy, then all leaves have finite volume and finite holonomy group.

Compare [2] for the above two corollaries.

Corollary 4. Let $L$ be a leaf with finite holonomy group. Then $\operatorname{gr}(\mathcal{F})=$ $\operatorname{gr}(L)$.

See [7] or [5] for the basic properties of the growth of a leaf and the growth type of a foliation.

Corollary 3 can be restated in the following form.

THEOREM 5. Let $\mathcal{F}$ be a transversely analytical totally geodesic foliation. Then the growth type of the foliation is realized by the growth type of any leaf with finite holonomy group.

Final remark. The "transversely analytical" assumption can be replaced by any condition ensuring that the holonomy transformations are determined by their jets (cf. e.g. [10], [12]).

\section{References}

[1] R. A. Blumenthal and J. J. Hebda, De Rham decomposition theorem for foliated manifolds, Ann. Inst. Fourier (Grenoble) 33 (1983), 183-198.

[2] - - - Complementary distributions which preserve the leaf geometry and applications to totally geodesic foliations, Quart. J. Math. Oxford 35 (1984), 383-392.

[3] - , - Ehresmann connections for foliations, Indiana Univ. Math. J. 33 (1984), 597-611.

[4] G. Cairns, Feuilletages géodésiques, thèse, Université du Languedoc, Montpellier, 1987.

[5] G. Hector and U. Hirsch, Introduction to the Geometry of Foliations, Parts A and B, Vieweg, Braunschweig, 1981, 1983.

[6] D. L. Johnson and L. B. Whitt, Totally geodesic foliations, J. Differential Geom. 15 (1980), 225-235. 
[7] J. Plante, Foliations with measure preserving holonomy, Ann. of Math. 102 (1975), 327-361.

[8] H. Winkelnkemper, The graph of a foliation, Ann. Global Anal. Geom. 1 (1983), 51-75.

[9] - The number of ends of the universal leaf of a Riemannian foliation, in: Differential Geometry, Proc., Special Year, Maryland 1981-82, R. Brooks (ed.), Birkhäuser, 1983, 247-254.

[10] R. A. Wolak, Foliations admitting transverse systems of differential equations, Compositio Math. 67 (1988), 89-101.

[11] —, Le graphe d'un feuilletage admettant un système d'équations différentielles, Math. Z. 201 (1989), 177-182.

[12] —, Geometric Structures on Foliated Manifolds, Universidad de Santiago de Compostela, 1989 .

INSTITUTE OF MATHEMATICS

JAGIELLONIAN UNIVERSITY

REYMONTA 4

30-059 KRAKÓW, POLAND

Reçu par la Rédaction le 12.10.1993

Added in proof. In [0] the authors proved that the graph of a foliation without vanishing cycles is Hausdorff. It is not difficult to check a totally geodesic foliation has no vanishing cycles, thus its graph is always a Hausdorff manifold, and our assumption of "transverse analyticity" is superfluous.

[0] P. Dazord et G. Hector, Intégration symplectique des variétés de Poisson totalement asphériques, in: Symplectic Geometry, Groupoids and Integrable Systems, MSRI Lecture Notes 20, 1991, 37-72. 